LUCAs Melo NeVES ${ }^{1}$

Ana Claudia de Souza Fortaleza

FABRÍCIO EDUARDO ROSSI ${ }^{2}$

TieGO ApARECIDO DINIZ ${ }^{2}$

Malena Ricci PICOlO ${ }^{3}$

CAMILA BuONANI ${ }^{4}$

LEANDRO FERREIRA ${ }^{5}$

ISMAEL ForTE Freitas JÚNIOR ${ }^{4}$

\title{
Avaliação da aptidão funcional através do conjunto de testes da AAHPERD em mulheres na pós-menopausa: existe declínio entre a quinta e sexta década de vida?
}

\author{
Assessment of functional fitness through the set of \\ AAHPERD tests in women after menopause: \\ Is there a decline between the fifth and sixth decades of life?
}

Artigo Original

Palavras-chave

Pós-menopausa

Aptidão física

Avaliação

Exercício

Humanos

Efeito idade

Keywords

Postmenopause

Physical fitness

Evaluation

Exercise

Humans

Age effect

\section{Resumo}

OBJETIVO: analisar o nível de aptidão funcional de um grupo de mulheres na pós-menopausa da cidade de Presidente Prudente por meio do conjunto de testes de aptidão funcional da American Aliance for Health, Physical Education, Recreation and Dance e verificar se existe diferença entre grupos de mulheres na quinta e sexta década de vida. MÉTODOS: estudo de característica transversal, com 175 mulheres na pós-menopausa (dosagem de hormônio folículo estimulante $>26,72 \mathrm{mlU} / \mathrm{L}$ ) desenvolvido na cidade de Presidente Prudente no ano de 2013. Os critérios de inclusão foram não ter feito parte de nenhum tipo de intervenção motora sistematizada durante, pelo menos, seis meses do período que antecedeu a coleta de dados da investigação; não apresentar comprometimentos motores ou cognitivos que inviabilizassem a realização dos protocolos de avaliação, não apresentar doença crônica ou degenerativa, lesão musculo-esquelética ou comorbidade que pudessem impedir ou limitar a realização das avaliações. As avaliações foram conduzidas pelos mesmos avaliadores treinados. $\bigcirc$ grupo 50 a 59 anos apresentou valores médios para idade

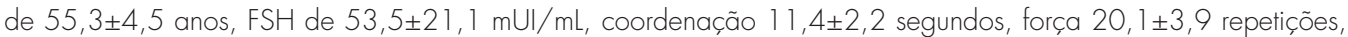
flexibilidade $51,7 \pm 1$ 1,8 centímetros, agilidade 23,2 $\pm 2,8$ segundos e Resistência aeróbia $500 \pm 43$ segundos e o grupo

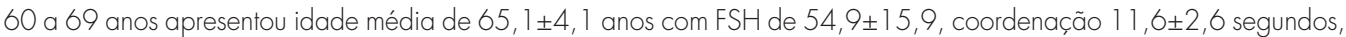

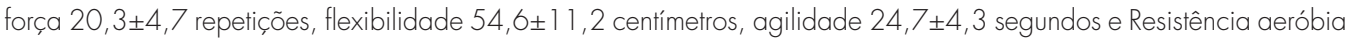
$508 \pm 51$ segundos. CONCLUSÃO: foi possível analisar a aptidão funcional de mulheres na pós-menopausa por meio do conjunto de testes da American Aliance for Health, Physical Education, Recreation and Dance não havendo diferenças significativas para as variáveis força, flexibilidade, capacidade aeróbia e coordenação, sendo que apenas a variável agilidade demonstrou diferenças significativas. Recomenda-se outros estudos, buscando formular valores normativos para a população em questão.

\section{Abstract}

PURPOSE: to analize the level of functional fitness of a group of postmenopausal women in the city of Presidente Prudente using the set of functional fitness tests of the American Alliance for Health, Physical Education, Recreation and Dance and to check whether there are differences between groups of women in the fifth and sixth decade of life. METHODS: This was a cross-sectional study conducted on 175 postmenopausal women (follicle stimulating hormone level>26.72 $\mathrm{mlU} / \mathrm{L}$ ) in the city of Presidente Prudente in 2013. The inclusion criteria were not being part of any type of systematic motor intervention for at least six months before the collection of research data; absence of motor or cognitive impairment that would prevent the evaluation protocols, and absence of chronic or degenerative disease, musculoskeletal injury or comorbidity that could prevent or limit the evaluations. The women were evaluated by the same trained examiners. The 50 to 59 year group showed a mean age of $55.3 \pm 4.5$ years, mean FSH values of $53.5 \pm 21.1 \mathrm{mlU} / \mathrm{mL}$, mean coordination of $11.4 \pm 2.2$ seconds, mean strength of $20.1 \pm 3.9$ repetitions, mean flexibility of $51.7 \pm 11.8 \mathrm{~cm}$,

Correspondência

Lucas Melo Neves

Centro de Estudos e Laboratório de Avaliação e Prescrição de Atividades Motoras - CELAPAM - Departamento de Educacão

Física - Universidade Estadual Paulista Rua Roberto Simonsen, 305 - Centro Educaciono CEP: $19060-900$

Presidente Prudente (SP), Brasil

Recebido 04/03/2015
Centro de Estudos e Laboratório de Avaliação e Prescrição de Atividades Motoras - CELAPAM - Departamento de Educação Física Universidade Estadual Paulista "Júlio de Mesquita Filho" - UNESP - Presidente Prudente (SP), Brasil.

Programa de Pós-Graduação em Biodinâmica do Movimento Humano, Universidade de São Paulo - USP - São Paulo (SPI, Brasil. 2Programa de Pós Graduação em Ciências da Motricidade, Universidade Estadual Paulista "Júlio de Mesquita Filho" - UNESP - Rio Claro (SP), Brasil.

${ }^{3}$ Centro de Estudos e Laboratório de Avaliação e Prescrição de Atividades Motoras, Universidade Estadual Paulista "Júlio de Mesquita Filho" - UNESP - Presidente Prudente (SP), Brasil.

${ }^{4}$ Departamento de Educação Física, Universidade Estadual Paulista "Júlio de Mesquita Filho" - UNESP - Presidente Prudente (SPI, Brasil ${ }^{5}$ Centro Universitário UNIFAFIBE, Bebedouro (SP), Brasil; Centro Universitário da Fundação Educacional de Barretos - Barretos (SP), Brasil. Conflito de interesses: não há. 
mean $23.2 \pm 2.8$ seconds agility and mean aerobic resistance of $500 \pm 43 / 2$. The 60 to 69 year group had a mean age of $65.1 \pm 4.1$ years with FSH $54.9 \pm 15.9,11.6 \pm 2.6$ seconds coordination, strength $20.3 \pm 4.7$ repetitions, $54.6 \pm 11.2 \mathrm{~cm}$ flexibility, agility $24.7 \pm 4.3$ seconds, and aerobic resistance of $508 \pm 51$ seconds. CONCLUSION: It was possible to analyze the functional fitness of postmenopausal women through the set of the American Alliance testing for Health, Physical Education, Recreation and Dance with no significant differences between groups for the variables strength, flexibility, aerobic capacity and coordination, and with only the speed variable showing significant differences. We recommend further studies seeking to formulate normative values for the population in question.

\section{Introdução}

Aptidão funcional é definida como a capacidade física do indivíduo às demandas comuns e inesperadas da vida diária de forma segura e eficaz ${ }^{1}$. Sua avaliação se torna relevante em adultos após os 50 anos, visto o declínio em relação a aptidão funcional observado a partir dessa idade, como perda de força muscular, níveis de condicionamento aeróbio, agilidade, equilíbrio, entre outros fatores ${ }^{2,3}$. Tais variáveis têm sido aferidas através de diversos instrumentos que podem ser didaticamente divididas em 3 grupos: testes estabelecidos por uma instituição, como o proposto pela American Aliance for Health, Physical Education, Recreation and Dance (AAHPERD); conjunto de testes proposto por pesquisadores independentes, como a Senior Fitness Test ${ }^{4}$; ou uso de diversas avaliações distintas em relação as capacidades físicas que compõe um conjunto de testes como observado em estudos recentes ${ }^{5,6}$. Especificamente no conjunto de testes proposto pela AAHPERD, verifica-se um grande número de estudos em idosos ${ }^{7-12}$ e seus detalhamentos são encontrados em alguns estudos tanto de conceituação da metodologia ${ }^{1}$ como na aplicação da mesma ${ }^{13,14}$.

A metodologia proposta pela AAHPERD tem sido amplamente utilizada em idosos brasileiros, tanto em mulheres quanto em homens ${ }^{15-19}$. Considerando apenas estudos publicados em periódicos indexados na plataforma Scielo, verificamos estudos de análises transversais de grupos específicos como idosas ativas ${ }^{15}$ e homens entre 60 e 69 anos $^{17}$, além de estudos que verificam a relação entre peso corporal e desempenho em idosas ${ }^{18}$. Estudos em outras plataformas como a PubMed, especialmente estudos de intervenção, também tem explorado tal metodologia ${ }^{7,9,11}$. Porém, apesar de sua utilização ampla, estudos de base considerando amostras representativas de acordo com conceitos estatísticos conhecidos, ainda não foram apontados na literatura, sendo apenas descritos estudos com grupos pequenos não representativos da população usada como objeto de estudo com percentis dos mesmos ${ }^{17}$.

Mesmo com as limitações quanto a representatividade da população nos estudos citados, verificamos uma lacuna em relação a estudos com a população de mulheres na pós-menopausa, visto que as pesquisas aqui citadas não consideram essa particularidade. Assim, a verificação das variáveis tratadas no conjunto de testes da AAHPERD pode ser um importante indicativo de autonomia e dos níveis de aptidão física de mulheres na pós-menopausa, como a verificação se existe declínio quando comparados mulheres entre 50 e 59 anos e 60 e 69 anos.

\section{Métodos}

Trata-se de um estudo de característica transversal, desenvolvido no Centro de Estudos e Laboratório de Avaliação e Prescrição de Atividades Motoras (CELAPAM) no Departamento de Educação Física, Faculdade de Ciências e Tecnologia (FCT) da Universidade do Estado de São Paulo (UNESP), Campus de Presidente Prudente, Brasil. A população escolhida para o estudo foi composta por mulheres na pós-menopausa (dosagem de $\mathrm{FSH}>26,72 \mathrm{mIU} / \mathrm{L}$ ). As participantes foram recrutadas e avaliadas no ano de 2013 por meio de jornais, rádios e televisão em forma de convite para participarem do estudo. Para inclusão no estudo, as voluntárias não deveriam ter participado de nenhum tipo de programa de exercícios físicos nos seis meses anteriores ao início da coleta de dados; não apresentar comprometimentos motores ou cognitivos; não apresentar doença crônica ou degenerativa, lesão músculo-esquelética ou qualquer comorbidade que pudessem impedir ou limitar a realização das avaliações.

O presente estudo obteve aprovação do Comitê de Ética em Pesquisa FCT-UNESP Presidente Prudente, Brasil (Certificado de Apresentação para Apreciação Ética - CAAE $\mathrm{n}^{\circ}$ 11547013.2.0000.5402). Todas as participantes que concordaram em fazer parte da investigação assinaram o Termo de Consentimento Livre e Esclarecido e a pesquisa foi conduzida de acordo com a Declaração de Helsinque revisada em 2008.

\section{Seleção da Amostra e delineamento do estudo}

Participaram do estudo 175 mulheres na pós-menopausa que foram distribuídas em dois grupos, quais sejam: grupo 50 a 59 anos ( $n=99)$ e grupo 60 a 69 anos $(n=76)$. A seleção da amostra foi realizada após triagem, considerando os critérios de inclusão acima citados. Todas as voluntárias participaram de uma entrevista conduzida por avaliadores previamente treinados que também realizaram os testes de aptidão funcional. Avaliação de níveis de FSH foi realizada em um laboratório de análises clínicas da cidade de Presidente Prudente. 


\section{Avaliações e descrição dos testes}

O conjunto de testes utilizado para avaliação da aptidão funcional foi proposto pela AAHPERD e consiste de cinco testes motores. Os parâmetros avaliados neste conjunto de testes têm estreita relação com a independência funcional do indivíduo, sendo que havendo um melhor desempenho haverá maior autonomia. As variáveis avaliadas de forma indireta são: agilidade e equilíbrio dinâmico, coordenação, flexibilidade, resistência de força e a resistência aeróbia geral ${ }^{1}$.

Para o teste de agilidade e equilíbrio dinâmico; o participante inicia o teste sentando em uma cadeira com os calcanhares no solo, sendo que a sua direita e a sua esquerda se encontra um cone posicionado a 1,50 m para trás e 1,80 m para o lado da cadeira. Ao sinal do avaliador, o avaliado move-se para a direita, contorna o cone, retorna e senta-se, elevando os pés, retirando-os do solo, para que o avaliador certifique-se de que o avaliado encontra-se realmente sentado. Imediatamente, o participante levanta-se novamente e move-se para a esquerda realizando o mesmo procedimento anterior. O teste consiste em contornar os cones da direita, da esquerda, da direita e da esquerda, novamente, totalizando 4 deslocamentos. São realizadas duas tentativas e o menor tempo é anotado em segundos como resultado final.

Para o teste de coordenação, um pedaço de fita adesiva com 76,2 cm de comprimento é fixado sobre uma mesa. Sobre a fita são feitas 6 marcas com 12,7 cm equidistantes entre si com a primeira e última marca a $6,36 \mathrm{~cm}$ de distância das extremidades da fita. Sobre cada uma das 6 marcas é afixado, perpendicularmente à fita, um outro pedaço de fita adesiva com $7,6 \mathrm{~cm}$ de comprimento. $\mathrm{O}$ participante senta-se de frente para a mesa e usa sua mão dominante para realizar o teste. Se a mão dominante for a direita, uma primeira lata de refrigerante é colocada marca 1 que corresponde a posição 1 , uma segunda lata na marca três (posição 3) e a terceira lata na marca 5 (posição 5). A mão direita é colocada na lata 1, com o polegar para cima, estando o cotovelo flexionado num ângulo de 100 a 120 graus. Quando o avaliador sinalizar, inicia-se o teste cronometrado, devendo o participante virar a lata, invertando sua base de apoio, de forma que a lata 1 seja colocada na posição 2, a lata 2 na posição 4, e a lata 3 na posição 6 . Em seguida, o avaliado, agora estando com o polegar apontado para baixo, apanha a lata 3 e inverte novamente sua base, recolocando-a na posição 5 , e da mesa forma, procede com as outras latas. Caso o participante seja canhoto, o mesmo procedimento é adotado, mas com as latas colocadas a partir da esquerda. São concedidas duas tentativas para a prática, e em seguida duas tentativas válidas para avaliação. Nestas duas últimas é anotada e o menor tempo é considerado como resultado final.

Para avaliação da flexibilidade; o participante sentado no solo, com as pernas estendidas, se posiciona sobre a marca zero de uma fita métrica afixada no solo. À marca de $63,5 \mathrm{~cm}$ de distância em relação à marca zero da fita, é afixada perpendicularmente uma fita adesiva que indica a posição dos calcanhares que devem estar $15,2 \mathrm{~cm}$ equidistantes do centro da fita métrica. Então, com as mãos uma sobre a outra, o participante é orientado a deslizá-las vagarosamente sobre a fita métrica tão distante quanto pode, permanecendo na posição final no mínimo por dois segundos. São oferecidas duas tentativas da prática, seguidas de duas tentativas de teste. O resultado final é dado pela melhor das duas tentativas anotadas.

Para a avaliação da resistência de força, é utilizado um halter pesando $2 \mathrm{~kg}$. O participante senta em uma cadeira sem braços, apoiando as costas no encosto da cadeira, com o tronco ereto, olhando para frente e com a planta dos pés completamente apoiados no solo. O braço dominante deve permanecer relaxado e estendido ao longo do corpo, enquanto a mão não dominante apoia-se sobre a coxa. O primeiro avaliador se posiciona ao lado do avaliado, colocando uma mão sobre o seu bíceps e a outra suporta o halter que é colocado na mão dominante do participante. O halter deve estar paralelamente ao solo com uma de suas extremidades voltadas para frente. Quando o segundo avaliador responsável pelo cronometro sinalizar o início do teste, o participante contrai o bíceps, realizando uma flexão do cotovelo até que o antebraço toque a mão do primeiro avaliador, que está posicionada no bíceps do avaliado. Após esse tempo, o teste é reiniciado, repetindo-se o mesmo procedimento, com a diferença de que o avaliado realiza o maior número de repetições no tempo de 30 segundos, sendo anotado como resultado final do teste o melhor desempenho de duas tentativas realizadas.

Finalmente, para avaliação da capacidade aeróbia geral, o participante é orientado para caminhar o mais rápido possível $804,67 \mathrm{~m}$, sem correr, em uma pista de atletismo de 400 metros. O tempo é anotado em minutos e segundo e reduzidos a segundos.

A avaliação dos níveis de FSH foi realizada em laboratório privado da cidade de Presidente Prudente, com experiência em atendimento à população local e regional. Outras análises que não são objetivo dessa pesquisa também foram realizadas, sendo as voluntárias orientadas a não realizar atividade física no dia anterior a coleta, manter jejum por 12 horas, e se apresentarem entre $7 \mathrm{~h} \mathrm{e} 7 \mathrm{~h}$ 30 min da manhã na unidade do respectivo laboratório.

As características dos grupos incluídos quanto a idade, peso, altura, índice de massa corporal (IMC) e nível de escolaridade são apresentadas na Tabela 1 .

\section{Análise dos dados}

Para a apresentação dos resultados, foi utilizada análise descritiva, com dados de média e desvio padrão. Foi realizado também teste de normalidade dos dados 
considerando os grupos 50 a 59 anos e 60 a 69 anos, bem como teste $t$ de Student para variáveis independentes e teste de Mann Whitney para variável dependente (somente variável agilidade) para verificação de diferenças entre os grupos. Todas as análises foram realizadas no software SPSS versão 17.0 .

\section{Resultados}

Na Tabela 2 são apresentados os resultados dos grupos 50 a 59 anos e 60 a 69 anos para as variáveis de aptidão funcional, bem como a idade e valores de FSH.

As pacientes do grupo 50 a 59 anos apresentaram média de idade de 55,3 $\pm 4,5$ anos com FSH de 53,5 $\pm 21,1 \mathrm{mUI} / \mathrm{mL}$ e o grupo 60 a 69 anos apresentou média de idade de $65,1 \pm 4,1$ anos com FSH de 54,9 $\pm 15,9$. Não houve diferenças significativas entre os grupos quanto aos níveis de FSH $(p=0,15)$. Na avaliação do conjunto de testes motores da AAHPERD, as variáveis: coordenação, força, flexibilidade e resistência aeróbia não foi observado diferenças significativas. Para a variável coordenação o valor médio para o grupo 50 a 59 anos foi de $11,4 \pm 2$,2 segundos e para o grupo 60 a 69 anos de 11,6 2 2,6 segundos $(\mathrm{p}=0,7)$. Quanto à variável força, o valor médio do grupo 50 a 59 anos foi de $20,1 \pm 3,9$ repetições e no grupo 60 a 69 anos de 20,3 $\pm 4,7$ repetições $(p=0,8)$. No teste de flexibilidade, para o grupo 50 a 59 anos, observou-se média de $51,7 \pm 11,8$ centímetros, e no grupo 60 a

Tabela 1. Características amostrais quanto a idade, peso, altura, índice de massa corporal e nível de escolaridade da amostra analisada

\begin{tabular}{|c|c|c|}
\hline Variáveis & $\begin{array}{c}50 \text { a } 59 \text { anos ( } \mathrm{n}=99) \\
\text { Média } \pm \mathrm{DP}\end{array}$ & $\begin{array}{c}60 \text { a } 69 \text { anos }(\mathrm{n}=76) \\
\text { Média } \pm \mathrm{DP}\end{array}$ \\
\hline Idade (anos) & $55,3 \pm 4,5$ & $65,1 \pm 4,1$ \\
\hline Peso (kg) & $69,3 \pm 16,8$ & $68,6 \pm 14,5$ \\
\hline Altura $(\mathrm{cm})$ & $161,0 \pm 18,0$ & $163,0 \pm 17,0$ \\
\hline IMC $\left(\mathrm{kg} / \mathrm{m}^{2}\right)$ & $28,2 \pm 3,2$ & $28,4 \pm 3,0$ \\
\hline Nível de escolaridade (anos) & $10,4 \pm 4,1$ & $10,5 \pm 4,3$ \\
\hline
\end{tabular}

IMC: índice de massa corporal.

Tabela 2. Valores observados na aptidão funcional entre os grupos de 50 a 59 anos e 60 a 69 anos

\begin{tabular}{|c|c|c|c|}
\hline Variáveis & $\begin{array}{c}50 \text { a } 59 \text { anos } \\
\text { (n=99) } \\
\text { Média } \pm D P\end{array}$ & $\begin{array}{c}60 \text { a } 69 \text { anos } \\
(\mathrm{n}=76) \\
\text { Média } \pm \mathrm{DP}\end{array}$ & Valor $\mathbf{p}$ \\
\hline Idade (anos) & $55,3 \pm 4,5$ & $65,1 \pm 4,1$ & $<0,001$ \\
\hline FSH (mUI/mL) & $53,5 \pm 21,1$ & $54,9 \pm 15,9$ & 0,154 \\
\hline Coordenação (segundos) & $11,4 \pm 2,2$ & $11,6 \pm 2,6$ & 0,717 \\
\hline Força (repetições) & $20,1 \pm 3,9$ & $20,3 \pm 4,7$ & 0,881 \\
\hline Flexibilidade (cm) & $51,7 \pm 11,8$ & $54,6 \pm 11,2$ & 0,058 \\
\hline Agilidade (segundos) & $23,2 \pm 2,8$ & $24,7 \pm 4,3$ & $0,028 *$ \\
\hline $\begin{array}{l}\text { Resistência aeróbia } \\
\text { (segundos) }\end{array}$ & $500,0 \pm 43,0$ & $508,0 \pm 51,0$ & 0,351 \\
\hline
\end{tabular}

FSH: hormônio folículo-estimulante.
69 anos de 54,6 $\pm 11,2$ centímetros ( $p=0,05)$; e, por fim, no teste de resistência aeróbia, os resultados médios do grupo 50 a 59 anos foi de $500 \pm 43$ segundos e no grupo 60 a 69 anos foi de $508 \pm 51$ segundos $(\mathrm{p}=0,3)$. Apenas quanto à variável agilidade houve diferenças significativas entre os grupos com resultados médios do grupo 50 a 59 anos de $23,2 \pm 2,8$ segundos e no grupo 60 a 69 anos de $24,7 \pm 4,3$ segundos $(\mathrm{p}=0,02)$.

\section{Discussão}

A análise transversal da aptidão funcional utilizando o conjunto de avaliações da AAHPERD foi possível, como apresentado, sendo esse o primeiro estudo disponível nas plataformas Scielo e PubMed no qual utilizou-se essa análise exclusivamente em mulheres na pós-menopausa. Os valores verificados estão próximos aos apresentados na literatura em estudos com mulheres em diversas faixas etárias. Em relação aos valores médios obtidos em cada teste, o grupo 50 a 59 anos apresentou melhor desempenho que o grupo 60 a 69 anos em todas as variáveis, com exceção da variável flexibilidade. Porém, apenas para a variável agilidade foi verificada diferença significante $\left(\mathrm{p}=0,028^{*}\right)$ entre o grupos 50 a 59 anos quando comparadas ao grupo 60 a 69 anos. Outras investigações publicadas previamente também analisaram o desempenho de aptidão funcional em mulheres no conjunto de testes da AAHPERD. No entanto, os estudos anteriores não discriminaram as voluntárias quanto ao tempo de menopausa.

Alguns pesquisadores têm explorado a importância da manutenção da aptidão funcional por meio do exercício físico ${ }^{15}$ e a influência da composição corporal na aptidão funcional ${ }^{18}$. Foi demonstrado que o excesso de peso pode contribuir negativamente com a aptidão funcional, sendo que idosas com excesso de peso têm chance cinco vezes maior de apresentar uma aptidão funcional fraca. Nesses casos, a prática regular de exercícios pode influenciar positivamente a aptidão funcional ao servir para controlar a composição corporal. Nosso estudo não se concentrou em verificar diferenças em relação a composição corporal, embora, em outras oportunidades, já tenhamos demonstrado a influência da composição corporal no desempenho da marcha ${ }^{20}$, fato associado a maior independência funcional. Porém, a verificação que apenas a variável agilidade se mostrou significante nos dados aqui publicados nos mostra que, apesar do declínio comumente associado ao envelhecimento, a aptidão funcional avaliada através do conjunto de testes de AAHPERD não apresenta diferenças entre mulheres na quinta década de vida comparada a sexta década de vida.

Algumas características importantes merecem ser discutidas em futuras investigações. O tempo em que as voluntárias estão na pós-menopausa, bem como a utilização 
de terapia de reposição hormonal podem ser associados ao desempenho funcional, visto não haver um consenso sobre a influência da reposição hormonal na aptidão funcional ${ }^{10,12}$. Delineamentos similares ao presente estudos podem ser realizados, principalmente comparando-se grupos ativos e sedentários, bem como mulheres na pós-menopausa e mulheres na fase fértil buscando verificar as influências dessas características. Essas questões carecem de investigação com base nos trabalhos até o momento já publicados.

Com base nos dados apresentados, concluímos que a utilização do conjunto de testes de aptidão funcional da American Aliance for Health, Physical Education, Recreation and Dance se fez possível para a avaliação da aptidão funcional em mulheres na menopausa, e que as capacidades força, flexibilidade, capacidade aeróbia e coordenação não apresentam diferenças significativas, sendo que apenas a variável agilidade demonstrou significância quando comparado grupos que estejam na quinta década (50 a 59 anos) de vida ao grupo sexta década (60 a 69 anos) de vida.

Sugerimos tal conjunto de testes para a avaliação da aptidão em mulheres na menopausa, cabendo a realização de novos estudos de base populacional para entender o perfil da população citada em relação a aptidão funcional, questão não respondida pelo presente estudo devido a limitações na seleção da amostra o número de envolvidos.

\section{Referências}

1. Osness WH, Adrian M, Clark B, Hoeger W, Raab D, Wisnell R. Functional fitness assessment for adults over 60 years (a field based assessment). Reston: American Alliance for Health. Physical Education Recreation and Dance; 1990.

2. Danneskiold-Samsoe B, Bartels EM, Bulow PM, Lund H, Stockmarr A, Holm CC, et al. Isokinetic and isometric muscle strength in a healthy population with special reference to age and gender. Acta Physiol (Oxf). 2009;197 Suppl 673:1-68.

3. Shore WS, DeLateur BJ. Prevention and treatment of frailty in the postmenopausal woman. Phys Med Rehabil Clin N Am. 2007;18(3):609-21, xii.

4. Rikli RE, Jones CJ. Functional fitness normative scores for communityresiding older adults, ages 60-94. J Aging Phys Act. 1999;7(2):162-81.

5. Cruz-Ferreira A, Marmeleira J, Formigo A, Gomes D, Fernandes J. Creative dance improves physical fitness and life satisfaction in older women. Res Aging. 2015. pii: 0164027514568103 . [Epub ahead of print].

6. Oppewal A, Hilgenkamp TI, van Wijck R, Schoufour JD, Evenhuis $\mathrm{HM}$. Physical fitness is predictive for a decline in daily functioning in older adults with intellectual disabilities: results of the HA-ID study. Res Dev Disabil. 2014;35(10):2299-315

7. da Silva RF, Sertorio JT, Lacchini R, Trapé AA, Tanus-Santos JE, Rush $J W$, et al. Influence of training status and eNOS haplotypes on plasma nitrite concentrations in normotensive older adults: a hypothesisgenerating study. Aging Clin Exp Res. 2014;26(6):591-8.

8. Goncalves LH, Silva AH, Mazo GZ, Benedetti TR, dos Santos SM, Marques $S$, et al. [Institutionalized elderly: functional capacity and physical fitness]. Cad Saúde Pública. 2010;26(9):1738-46. Portuguese.

9. Hernandez SS, Coelho FG, Gobbi S, Stella F. [Effects of physical activity on cognitive functions, balance and risk of falls in elderly patients with Alzheimer's dementia]. Rev Bras Fisioter. 2010;14(1):68-74. Portuguese.

10. Justino Borges L, Bertoldo Benedetti TR, Zarpellon Mazo G. [The influence of physical exercise on depressive symptoms and functional fitness in elderly residents of south Brazil]. Rev Esp Geriatr Gerontol. 2010;45(2):72-8. Spanish.
11. Trapé AA, Jacomini AM, Muniz JJ, Sertorio JT, Tanus-Santos JE, do Amaral SL, et al. The relationship between training status, blood pressure and uric acid in adults and elderly. BMC Cardiovasc Disord. 2013;13:44.

12. Wood RH, Reyes R, Welsch MA, Favaloro-Sabatier J, Sabatier $M$, Matthew Lee $C$, et al. Concurrent cardiovascular and resistance training in healthy older adults. Med Sci Sports Exerc. $2001 ; 33(10): 1751-8$

13. Benedetti TR, Mazo GZ, Gobbi S, Amorim M, Gobbi LT, Ferreira $L$, et al. Valores normativos de aptidão funcional em mulheres de 70 a 79 anos. Rev Bras Cineantropom Desempenho Hum. 2007;9(1):28-36.

14. Benedetti TR, Mazo GZ, Goncalves LH. Adaptation of the AAHPERD test battery for institutionalized older adults. Rev Bras Cineantropom Desempenho Hum. 2014;16(1):1-14.

15. Cipriani NC, Meurer ST, Benedetti TR, Lopes MA. Aptidão funcional de idosas praticantes de atividades físicas. Rev Bras Cineantropom Desempenho Hum. 2010;12(2):106-11.

16. Hoefelmann CP, Benedetti TR, Antes DL, Lopes MA, Mazo GZ, Korn S. Aptidão funcional de mulheres idosas ativas com 80 anos ou mais. Motriz Rev Educ Fís (Impr). 2011 ; 17(1):19-25.

17. Mazo GZ, Benedetti TR, Gobbi S, Ferreira L, Lopes MA. Valores normativos e aptidão funcional em homens de 60 a 69 anos de idade. Rev Bras Cineantropom Desempenho Hum. 2010;12(5):316-23.

18. Rech CR, Cruz JL, Araújo EDS, Kalinowski FG, Dellagrana RA. Associação entre aptidão funcional e excesso de peso em mulheres idosas. Motricidade. 2010;6(2):47-53.

19. Virtuoso Júnior JS, Guerra RO. Confiabilidade de testes de aptidão funcional em mulheres de 60 a 80 anos. Motricidade. $2011 ; 7(2): 7-13$.

20. Fortaleza AC, Rossi FE, Buonani C, Fregonesi CE, Neves $L M$, Diniz TA, et al. [Total body and trunk fat mass and the gait performance in postmenopausal women]. Rev Bras Ginecol Obstet. 2014;36(4):176-81. Portuguese. 